\title{
CODEBOOK DESIGN FOR NON-COHERENT COMMUNICATION IN MULTIPLE-ANTENNA SYSTEMS
}

\author{
Marko Beko*, João Xavier and Victor Barroso \\ Instituto Superior Técnico - Instituto de Sistemas e Robótica \\ Av. Rovisco Pais, 1049-001 Lisboa, Portugal \\ \{marko, jxavier, vab\}@isr.ist.utl.pt
}

\begin{abstract}
We address the problem of space-time codebook design for noncoherent communications in multiple-antenna wireless systems. The channel matrix is assumed deterministic (no stochastic model assumed) and unknown at both the receiver and the transmitter. In contrast with other approaches, the Gaussian observation noise has an arbitrary correlation structure, known by the transmitter and the receiver. To handle the unknown deterministic space-time channel, a GLRT receiver is implemented. We propose a new methodology for space-time codebook design under this non-coherent setup. This optimizes the probability of error of the receiver's detector in the high SNR regime, thus solving a high-dimensional nonlinear nonsmooth optimization problem in a two-step approach: (i) firstly, a convex SDP relaxation yields a rough estimate of the optimal codebook; (ii) this is then refined through a geodesic descent optimization algorithm that exploits the Riemannian geometry imposed by the power constraints on the space-time codewords. Computer simulations demonstrate that, for the specific case of spatio-temporal white observation noise, our codebooks are marginally better than those provided by state-of-art known solutions. However, the most relevant conclusion is that, for correlated noise environments, our method provides codes that significantly outperform other known codes.
\end{abstract}

\section{INTRODUCTION}

In slowly fading scenarios, channel stability enables the receiver to be trained in order to acquire the channel state information (CSI) necessary for coherent detection of the transmitted codeword. Specific codebook design techniques have been developed for coherent systems, relying on the availability of CSI at the receiver. It is known [1] that, for this kind of codes, the capacity of the multipleantenna link increases linearly (for rich scattering environments) with the minimum number of transmitters and receivers. In fast fading scenarios, channel stability is lost, CSI is no more available, and the receiver must then operate in a non-coherent mode.

Previous work. The capacity of non-coherent multiple systems was studied in [2,3]. Under the additive white noise and Rayleigh channel assumptions, it has been shown that the high reception SNR scenario is sufficient to guarantee that unitary constellations are capacity optimal. In $[4,5]$, a systematic method for designing unitary space-time constellations was presented. However, the techniques therein can not be readily extended to the correlated noise scenario,

\footnotetext{
* This work was supported by the FCT Programa Operacional Sociedade de Informação (POSI) in the frame of Quadro Comunitário de Apoio III, and
} by FCT PhD Grant SFRH/BD/12809/2003. under which it is unlike that unitary space-time constellations can be optimal.

Contribution. The main contribution of this paper is a new algorithm that systematically designs space-time codebooks for noncoherent multiple-antena communication systems. Contrary to other approaches, the Gaussian observation noise may have an arbitrary correlation structure. In this general case, computer simulations show that the space-time codes obtained with our method significantly outperform those already known.

Paper organization. In section 2, we formulate the problem addressed in this paper. We describe the non-coherent receiver and discuss the selection of the codebook design criterium. In section 3 we propose a new algorithm that systematically design non-coherent space-time constellations for arbitrary noise covariance matrix and any $M, N, K$ and $T$, respectively, number of transmitter antennas, number of receiver antennas, size of codebook, and channel coherence interval. In Section 4, we present codebook constructions for several important special cases and compare their performance with state-of-art solutions. Section 5 presents the main conclusions of our paper.

\section{PROBLEM FORMULATION}

Data model and assumptions. The communication system comprises $M$ transmit and $N$ receive antennas and we assume a block fading channel model with coherence interval $T$. In complex base band notation we have the model $\boldsymbol{Y}=\boldsymbol{X} \boldsymbol{H}^{H}+\boldsymbol{E}$, where $\boldsymbol{X}$ is the $T \times M$ matrix of transmitted symbols (the matrix $\boldsymbol{X}$ is called hereafter a space-time codeword), $\boldsymbol{Y}$ is the $T \times N$ matrix of received symbols, $\boldsymbol{H}$ is the $N \times M$ matrix of channel coefficients, and $\boldsymbol{E}$ is the $T \times N$ matrix of zero-mean additive observation noise. In $\boldsymbol{Y}$, time indexes the rows and space indexes the columns. We work under the following assumptions: (A1) The channel matrix $\boldsymbol{H}$ is not known at the receiver neither at the transmitter, and no stochastic model is assumed for it; (A2) The codeword $\boldsymbol{X}$ is chosen from a finite codebook $\mathcal{X}=\left\{\boldsymbol{X}_{1}, \boldsymbol{X}_{2}, \ldots, \boldsymbol{X}_{K}\right\}$ known to the receiver, where $K$ is the size of the codebook. We impose the power constraint $\operatorname{tr}\left(\boldsymbol{X}_{k}^{H} \boldsymbol{X}_{k}\right)=1$ for each codeword; (A3) The noise covariance matrix $\boldsymbol{\Upsilon}=\mathrm{E}\left[\operatorname{vec}(\boldsymbol{E}) \operatorname{vec}(\boldsymbol{E})^{H}\right]$ is known at the transmitter and at the receiver $(\operatorname{vec}(\boldsymbol{E})$ stacks all columns of the matrix $\boldsymbol{E}$ on the top of each other, from left to right). In assumption (A3), we let the data model depart from the customary assumption of spatiotemporal white Gaussian observation noise, which is clearly an approximation. In general, in realistic scenarios the $\boldsymbol{E}$ term may have very rich correlation structure, e.g, see pp.10,159,171 in [6]. The generalization to arbitrary noise covariance matrices $\Upsilon$ encompasses 
many scenarios of interest as special cases: spatially coloured or not jointly with temporally coloured or not observation noise, multiuser environment, etc.

Receiver. Under the above assumptions, the conditional probability density function of the received vector $\boldsymbol{y}=\operatorname{vec}(\boldsymbol{Y})$, given the transmitted matrix $\boldsymbol{X}$, is given by

$$
p(\boldsymbol{y} \mid \boldsymbol{X})=\frac{\exp \left\{-\left\|\boldsymbol{y}-\left(\boldsymbol{I}_{N} \otimes \boldsymbol{X}\right) \boldsymbol{g}\right\|_{\boldsymbol{\Upsilon}^{-1}}^{2}\right\}}{\pi^{T N} \operatorname{det} \boldsymbol{\Upsilon}},
$$

where $\boldsymbol{g}=\operatorname{vec}\left(\boldsymbol{H}^{H}\right)$ is the unknown realization of the channel, $\boldsymbol{I}_{N}$ is the $N \times N$ matrix and $\otimes$ denotes Kronecker product. Also, we use the notation $\|\boldsymbol{z}\|_{\boldsymbol{A}}^{2}=\boldsymbol{z}^{H} \boldsymbol{A} \boldsymbol{z}$.

Since no stochastic model is attached to the channel propagation matrix, the receiver faces a multiple hypothesis testing problem with the channel $\boldsymbol{H}$ as a deterministic nuisance parameter. We assume a generalized likelihood ratio test (GLRT) receiver which decides the index $k$ of the codeword as the index $\widehat{k}$ such that $\widehat{k}=$ $\operatorname{argmax}\left\{p\left(\boldsymbol{y} \mid \boldsymbol{X}_{k}, \widehat{\boldsymbol{g}}_{k}\right): k=1,2, \ldots, K\right\}$ where $\widetilde{\boldsymbol{X}_{k}}=\boldsymbol{I}_{N} \otimes \boldsymbol{X}_{k}$ and $\widehat{\boldsymbol{g}}_{k}=\left({\widetilde{\boldsymbol{X}_{k}}}^{H} \boldsymbol{\Upsilon}^{-1} \widetilde{\boldsymbol{X}}_{k}\right)^{-1} \widetilde{\boldsymbol{X}}_{k}{ }^{H} \boldsymbol{\Upsilon}^{-1} \boldsymbol{y}$. In words, the GLRT [7] consists in a bank of $K$ parallel processors where the $k$-th processor computes the likelihood of the observation assuming the presence of the $k$-th codeword and the channel is replaced by its ML estimate.

Codebook design criterion. In this paper, our goal is to design a codebook $\mathcal{X}=\left\{\boldsymbol{X}_{1}, \boldsymbol{X}_{2}, \ldots, \boldsymbol{X}_{K}\right\}$ of size $K$ for the current setup. A codebook $\mathcal{X}$ is a point in the space $\mathcal{M}=\left\{\left(\boldsymbol{X}_{1}, \ldots, \boldsymbol{X}_{K}\right)\right.$ : $\left.\operatorname{tr}\left(\boldsymbol{X}_{k}^{H} \boldsymbol{X}_{k}\right)=1\right\}$. Note that $\mathcal{M}$ can be viewed as multi-dimensional torus, i.e, the Cartesian product of $K$ unit-spheres. First, we must adopt a merit function $f: \mathcal{M} \rightarrow \mathbb{R}$ which gauges the quality of each constellation $\mathcal{X}$. The average error probability for a specific $\mathcal{X}$ would be the natural choice, but the theoretical analysis seems to be intractable. Instead, as usual [3], we rely on a pairwise error probability study to construct our merit function. For the special case of unitary codebooks $\left(\boldsymbol{X}_{k}^{H} \boldsymbol{X}_{k}=\frac{1}{M} \boldsymbol{I}_{M}\right)$ and spatio-temporal white Gaussian noise ( $\boldsymbol{\Upsilon}=\boldsymbol{I}_{T N}$ ) and iid Rayleigh fading, the exact expression and Chernoff upper bound for the pairwise error probability have been derived in [3]. However, the calculus of these expressions for the general case, i.e, arbitrary matrix constellations $\mathcal{X}$ and noise correlation matrix $\Upsilon \Upsilon$, seems to be burdensome. Instead, in this paper we resort to the asymptotic expression of the pairwise error probability in the high signal-to-noise-ratio (SNR) regime, for arbitrary $\mathcal{X}$ and $\boldsymbol{\Upsilon}$. Let $P_{\boldsymbol{X}_{i} \rightarrow \boldsymbol{X}_{j}}$ be the probability of the GLRT receiver deciding $\boldsymbol{X}_{j}$ when $\boldsymbol{X}_{i}$ is sent. It can be shown (details omitted) that

$$
\begin{aligned}
P_{\boldsymbol{X}_{i} \rightarrow \boldsymbol{X}_{j}} & \approx P\left(-2 \Re\left\{\boldsymbol{e}^{H} \boldsymbol{P}_{j}^{H} \boldsymbol{\Upsilon}^{-1} \boldsymbol{\Delta} \boldsymbol{g}\right\}>\boldsymbol{g}^{H} \boldsymbol{\Delta}^{H} \mathbf{\Upsilon}^{-1} \boldsymbol{\Delta} \boldsymbol{g}\right) \\
& =\mathcal{Q}\left(\frac{1}{\sqrt{2}} \sqrt{\boldsymbol{g}^{H} \boldsymbol{L}_{i j} \boldsymbol{g}}\right)
\end{aligned}
$$

where $\boldsymbol{P}_{i}=\boldsymbol{I}_{T N}-\widetilde{\boldsymbol{X}}_{i}\left(\widetilde{\boldsymbol{X}}_{i}^{H} \mathbf{\Upsilon}^{-1} \widetilde{\boldsymbol{X}}_{i}\right)^{-1} \widetilde{\boldsymbol{X}}_{i}^{H} \mathbf{\Upsilon}^{-1}, \boldsymbol{\Delta}=\boldsymbol{P}_{j} \widetilde{\boldsymbol{X}}_{i}$, $\boldsymbol{L}_{i j}=\widetilde{\boldsymbol{X}}_{i}{ }^{H} \boldsymbol{\Upsilon}^{-\frac{1}{2}} \boldsymbol{\Pi}_{j}^{+} \boldsymbol{\Upsilon}^{-\frac{1}{2}} \widetilde{\boldsymbol{X}}_{i}, \mathcal{Q}(x)=\int_{x}^{+\infty} \frac{1}{\sqrt{2 \pi}} e^{-\frac{t^{2}}{2}} d t, \boldsymbol{e}=$ $\operatorname{vec}(\boldsymbol{E}), \boldsymbol{\Pi}_{j}^{\perp}=\boldsymbol{I}_{T N}-\boldsymbol{\Upsilon}^{-\frac{1}{2}} \widetilde{\boldsymbol{X}}_{j}\left(\widetilde{\boldsymbol{X}}_{j}{ }^{H} \boldsymbol{\Upsilon}^{-1} \widetilde{\boldsymbol{X}}_{j}\right)^{-1} \widetilde{\boldsymbol{X}}_{j}{ }^{H} \boldsymbol{\Upsilon}^{-\frac{1}{2}}$ and $\Re\{x\}$ denotes the real part of complex number $x$. The proof is omitted due to paper length constraints (can be found in the companion paper [8]). Equation (1) shows that the probability of misdetecting $\boldsymbol{X}_{i}$ for $\boldsymbol{X}_{j}$, depends on the channel $\boldsymbol{g}=\operatorname{vec}\left(\boldsymbol{H}^{H}\right)$ and the relative geometry of the codewords $\widetilde{\boldsymbol{X}}_{i}$ and $\widetilde{\boldsymbol{X}}_{j}$. We can decouple the action of $\boldsymbol{g}$ and $\boldsymbol{L}_{i j}$ as follows: as $\boldsymbol{g}^{H} \boldsymbol{L}_{i j} \boldsymbol{g} \geq \lambda_{\min }\left(\boldsymbol{L}_{i j}\right)\|\boldsymbol{g}\|^{2}$ and $\mathcal{Q}(x)$ is monotonically non-increasing, we have the upper bound on the approximate pairwise error probability for high SNR

$$
P_{\boldsymbol{X}_{i} \rightarrow \boldsymbol{X}_{j}} \leq \mathcal{Q}\left(\frac{1}{\sqrt{2}}\|\boldsymbol{g}\| \sqrt{\lambda_{\min }\left(\boldsymbol{L}_{i j}\right)}\right) .
$$

We cannot control the power of the channel $\boldsymbol{g}=\operatorname{vec}\left(\boldsymbol{H}^{H}\right)$, but we can design codebooks aiming at maximizing $\lambda_{\min }\left(\boldsymbol{L}_{i j}\right)$.

Problem formulation. We are led to define the merit function $f$ : $\mathcal{M} \rightarrow \mathbb{R}, \mathcal{X}=\left\{\boldsymbol{X}_{1}, \ldots, \boldsymbol{X}_{K}\right\} \mapsto f(\mathcal{X})$ as $f(\mathcal{X})=\min \left\{f_{i j}(\mathcal{X}):\right.$ $1 \leq i \neq j \leq K\}$ where $f_{i j}(\mathcal{X})=\lambda_{\min }\left(\boldsymbol{L}_{i j}(\mathcal{X})\right)$. Constructing an optimal codebook $\mathcal{X}=\left\{\boldsymbol{X}_{1}, \boldsymbol{X}_{2}, \ldots, \boldsymbol{X}_{K}\right\}$ amounts to solving the following optimization problem

$$
\mathcal{X}^{*}=\underset{\mathcal{X} \in \mathcal{M}}{\arg \max } f(\mathcal{X})
$$

The problem defined in (2) is a high-dimensional, non-linear and non-smooth optimization problem. As an example, for a codebook of size $K=256$ the number of $f_{i j}$ functions is $K(K-1) \approx$ 62500. Also, for $T=8$ and $M=2$, there are $2 K T M=8192$ real variables to optimize. Moreover, note that we have

$$
f\left(\boldsymbol{X}_{1}, \boldsymbol{X}_{2}, \ldots, \boldsymbol{X}_{K}\right)=f\left(\boldsymbol{X}_{1} e^{i \theta_{1}}, \boldsymbol{X}_{2} e^{i \theta_{2}}, \ldots, \boldsymbol{X}_{K} e^{i \theta_{K}}\right)
$$

for any $\theta_{k} \in \mathbb{R}$ and $k=1, \ldots, K$. This means that $f$ depends on each $\boldsymbol{X}_{k}\left(\left\|\boldsymbol{X}_{k}\right\|=1\right)$ only through the line spanned by it (i.e., $\left\{\lambda \boldsymbol{X}_{k}: \lambda \in \mathbb{C}\right\}$ ). Thus, we can interpret the optimization problem in (2) as a packing problem in a product of projective spaces $[5,12]$.

\section{CODEBOOK CONSTRUCTION}

We propose a two-phase methodology to tackle the optimization problem in (2). In phase one, we start by solving a convex semidefinite programming (SDP) relaxation to obtain a rough estimate of the optimal codebook. Phase two refines it through a geodesic descent optimization algorithm (GDA) which efficiently exploits the Riemannian geometry of the constraints.

Phase 1: SDP relaxation. This phase constructs a sub-optimal codebook $\mathcal{X}^{*}=\left\{\boldsymbol{X}_{1}^{*}, \ldots, \boldsymbol{X}_{K}^{*}\right\}$. The codebook is constructed incrementally. There are several strategies for choosing first codeword $\boldsymbol{X}_{1}^{*}$, e.g, randomly generated, filling columns of the matrix with eigenvectors associated to the smallest eigenvalues of the noise covariance matrix,etc. We have found them equally efficient. Addition of a new codeword consists in solving a SDP. Let $\mathcal{X}_{k-1}^{*}=$ $\left\{\boldsymbol{X}_{1}^{*}, \ldots, \boldsymbol{X}_{k-1}^{*}\right\}$ be the codebook at the $k-1 \frac{t h}{\underline{t a}}$ stage. The new codeword is found by solving

$$
\begin{aligned}
& \boldsymbol{X}_{k}^{*}=\arg \max \quad f\left(\boldsymbol{X}_{1}^{*}, \ldots, \boldsymbol{X}_{k-1}^{*}, \boldsymbol{X}_{k}\right) \\
& \operatorname{tr}\left(\boldsymbol{X}_{k}^{H} \boldsymbol{X}_{k}\right)=1 \\
& =\underset{\operatorname{tr}\left(\boldsymbol{X}_{k}^{H} \boldsymbol{X}_{k}\right)=1}{\arg \max } \min _{1 \leq i \leq k-1}\left\{\lambda_{\min }\left(\boldsymbol{L}_{i k}\right), \lambda_{\min }\left(\boldsymbol{L}_{k i}\right)\right\}
\end{aligned}
$$

We can show (details omitted, see [8]) that the optimization problem defined in (3) is equivalent to

$$
\left(\boldsymbol{Y}^{*}, \widetilde{\boldsymbol{X}}^{*}, t^{*}\right)=\arg \max t
$$

with the following constraints

$$
\left[\begin{array}{ccc}
\operatorname{tr}\left(\boldsymbol{N}_{i} \boldsymbol{A}_{1} \boldsymbol{Y} \boldsymbol{B}_{1}\right)-t & \cdots & \operatorname{tr}\left(\boldsymbol{N}_{i} \boldsymbol{A}_{M N} \boldsymbol{Y} \boldsymbol{B}_{1}\right) \\
\vdots & & \vdots \\
\operatorname{tr}\left(\boldsymbol{N}_{i} \boldsymbol{A}_{1} \boldsymbol{Y} \boldsymbol{B}_{M N}\right) & \cdots & \operatorname{tr}\left(\boldsymbol{N}_{i} \boldsymbol{A}_{M N} \boldsymbol{Y} \boldsymbol{B}_{M N}\right)-t
\end{array}\right] \succeq \mathbf{0}
$$




$$
\begin{gathered}
{\left[\begin{array}{ll}
\boldsymbol{M} & \boldsymbol{Z}_{i} \\
\boldsymbol{Z}_{i}^{H} & \boldsymbol{P}_{i}
\end{array}\right] \succeq \mathbf{0} \forall_{1 \leq i \leq k-1}, \boldsymbol{S} \boldsymbol{Y} \boldsymbol{S}^{H}=\boldsymbol{K} \widetilde{\boldsymbol{X}} \boldsymbol{K}^{H}, \operatorname{tr}(\widetilde{\boldsymbol{X}})=1,} \\
\boldsymbol{A} \boldsymbol{Y} \boldsymbol{A}^{H}=1, \boldsymbol{Y}=\boldsymbol{Y}^{H}, \boldsymbol{Y} \succeq \mathbf{0}, \operatorname{rank}(\boldsymbol{Y})=1
\end{gathered}
$$

where $\widetilde{\boldsymbol{X}}=\operatorname{vec}\left(\boldsymbol{X}_{k}\right) \operatorname{vec}^{H}\left(\boldsymbol{X}_{k}\right), b^{2}=1$ and $\boldsymbol{Y}=\boldsymbol{z} \boldsymbol{z}^{H}$ with $\boldsymbol{z}=\left[\operatorname{vec}^{T}\left(\widetilde{\boldsymbol{X}_{k}}\right) b\right]^{T}$. The matrices $\boldsymbol{M}, \boldsymbol{Z}_{i}$ are linear in $\boldsymbol{Y}$, while $\boldsymbol{N}_{i}, \ddot{\boldsymbol{P}}_{i}, \boldsymbol{K}, \boldsymbol{S}, \boldsymbol{A}, \boldsymbol{A}_{i}$ and $\boldsymbol{B}_{i}$ are constants (not defined here due to the length constraint, but can be found in [8]). Because of the rank condition in (4), i.e. $\operatorname{rank}(\boldsymbol{Y})=1$, the design of the codewords, once again, translates into a high-dimensional difficult nonlinear optimization problem. However, ignoring this restriction the optimization problem becomes a convex one. The rank 1 relaxation is usually known as the Shor relaxation. Remark that for $K=256, M=2$, $N=2, T=8$ and in the last passage through the loop, i.e., for $k=K$, the output variable $\boldsymbol{Y}$ is of dimension $69 \times 69$ (does not depend on $K$ ) and the number of linear matrix inequality constraints that needs to be defined is of order $K$. To solve the optimization problem (4) we select the Self-Dual-Minimization package SeDuMi 1.1 [9]. Once the problem defined in (4) is solved we need to extract the $k^{\underline{t h}}$ codeword from the output variable $\widetilde{\boldsymbol{X}}$. Toward this end, we adopt a technique similar to [10]. The technique consists in generating independent realizations of vectors that obey Gaussian distribution with zero mean and covariance matrix $\widetilde{\boldsymbol{X}}$, i.e., $\boldsymbol{z}_{l} \stackrel{i i d}{\sim}$ $\mathcal{C N}(\mathbf{0}, \widetilde{\boldsymbol{X}})$, for $l=1,2, \ldots, L$, where $L$ is a parameter to be chosen. After forcing norm 1 , i.e., $\boldsymbol{v}_{l}=\boldsymbol{z}_{l} /\left\|\boldsymbol{z}_{l}\right\|$ for $l=1,2, \ldots, L$, we choose the $k-t h$ codeword, $\boldsymbol{X}_{k}^{*}=\operatorname{ivec}\left(\boldsymbol{v}_{l}^{*}\right)$ where

$$
l^{*}=\underset{l=1,2, \ldots, L}{\arg \max } f\left(\boldsymbol{X}_{1}^{*}, \boldsymbol{X}_{2}^{*}, \ldots, \boldsymbol{X}_{k-1}^{*}, \operatorname{ivec}(\boldsymbol{v})\right) .
$$

The operation "ivec" operates as an inverse of "vec" (reshapes the $T M$-dimensional vector into a $T \times M$ matrix). Note that $\boldsymbol{X}_{k}^{*}$ is a valid codeword because $\operatorname{tr}\left(\boldsymbol{X}_{k}^{* H} \boldsymbol{X}_{k}^{*}\right)=1$. We are clearly dealing with a suboptimal solution for a codebook.

Phase 2: Geodesic Descent Algorithm. Problem (4) requires the optimization of a non-smooth function over the smooth manifold $\mathcal{M}$. Phase 1 gives us a rough estimate of a codebook of size $K$. To refine this estimate we resort to an iterative algorithm, which we call GDA (geodesic descent algorithm). Due to the paper length constraint, we can only overview this iterative scheme. Let $\mathcal{X}_{k}$ be the $k^{\underline{t h}}$ iterate (the initialization $\mathcal{X}_{0}$ is furnished by phase 1 ). First, we identify the index set $\mathcal{A}$ of "active" constraint pairs (i,j), i.e., $\mathcal{A}=\left\{(i, j): f\left(\mathcal{X}_{k}\right)=f_{i j}\left(\mathcal{X}_{k}\right)\right\}$. Then, we check if there is an ascent direction $\Delta$ simultaneously for all functions $f_{i j}$ with $(i, j) \in \mathcal{A}$. This ascent direction $\Delta$ is searched within $T_{\mathcal{X}_{k}} \mathcal{M}$, the tangent space to $\mathcal{M}$ at $\mathcal{X}_{k}$, and consists in solving a linear program. If there are no such ascent direction, the algorithm stops. Otherwise, we perform an Armijo search for $f(\mathcal{X})$ along the geodesic which emanates from $\mathcal{X}_{k}$ in the direction $\Delta$. This Armijo search determines $\mathcal{X}_{k+1}$ and we repeat the loop. A geodesic is nothing but the generalization of a straight line in Euclidean space to a curved surface [11]. In loose terms, GDA resembles a sub-gradient method and consequently, the algorithm usually converges slowly near local minimizers. Note however that this is not a serious drawback since the codebooks are generated off-line.

\section{RESULTS}

We have constructed codes for three special categories of noise covariance matrices $\boldsymbol{\Upsilon}$. In all simulations we assumed a Rayleigh fading model for the channel matrix, i.e., $h_{i j} \stackrel{i i d}{\sim} \mathcal{C N}\left(0, \sigma^{2}\right)$. (1) The first category is spatio-temporal white observation noise, i.e., $\mathbf{\Upsilon}=$ $\mathrm{E}\left[\operatorname{vec}(\boldsymbol{E}) \operatorname{vec}(\boldsymbol{E})^{H}\right]=\boldsymbol{I}_{N T}$. We compared our codes with the best known found in [4]. We considered the case where the coherence interval $T=8, M=3$ transmit antennas, $N=1$ receive antennas and a codebook with $K=256$ codewords. In figure 1, we show the symbol error rate (SER) versus SNR $=\mathrm{E}\left\{\left\|\boldsymbol{X}_{k} \boldsymbol{H}^{H}\right\|^{2}\right\} / \mathrm{E}\left\{\|\boldsymbol{E}\|^{2}\right\}=$ $N \sigma^{2} / \operatorname{tr}(\mathbf{\Upsilon})$. The solid-plus and dashed-circle curves represent performances of codes constructed by our method, and unitary codes respectively. As we can see, our codebook construction is only marginally better for this particular case. For $M=1$, in table 1 we compare our results with [5] for $T=5$. We manage to improve the best known results and in some cases actually provide optimal packings which attain the Rankin upper bound. (2) The second category corresponds to spatially white-temporally coloured observation noise, i.e., $\boldsymbol{\Upsilon}=\boldsymbol{I}_{N} \otimes \Sigma(\boldsymbol{\rho})$ where the vector $\boldsymbol{\rho}: T \times 1$ is the first column of the Toeplitz matrix $\Sigma(\boldsymbol{\rho})$. In figures 2-3 the solid curves represent performance of codes constructed by our method, while the dashed curves represent performance of unitary codes. In either case, the plus sign indicates that the GLRT receiver is implemented. The square sign indicates that the Bayesian receiver is implemented (takes in account the statistics of the channel). Figure 2 plots the result of the experiment for $T=8, M=2, N=1, K=67$ and $\rho=[1 ; 0.85 ; 0.6 ; 0.35 ; 0.1 ; \operatorname{zeros}(3,1)]$. It can be seen that for SER $=10^{-3}$, our codes demonstrate a gain of $3 \mathrm{~dB}$ when compared with the unitary codes. (3) In the third category, we considered the case where the noise matrix is of the form $\boldsymbol{E}=\boldsymbol{s} \boldsymbol{\alpha}^{T}+\boldsymbol{E}_{\text {temp. }}$. This models an interfering source $s$ (with known covariance matrix $\Upsilon_{s}$ ) where the complex vector $\boldsymbol{\alpha}$ is the known channel attenuation between each receive antenna and the interfering source. The matrix $\boldsymbol{E}_{\text {temp }}$ has a noise covariance matrix belonging to the second category. Thus, the noise covariance matrix is given by $\Upsilon=$ $\boldsymbol{\alpha} \boldsymbol{\alpha}^{H} \otimes \boldsymbol{\Upsilon}_{s}+\boldsymbol{I}_{N} \otimes \Sigma(\boldsymbol{\rho})$. Figure 3 plots the result of the experiment for $T=8, M=2, N=2, K=32, s=[1 ; 0.7 ; 0.4 ; 0.15 ; \operatorname{zeros}(4,1)], \boldsymbol{\rho}=$ $[1 ; 0.8 ; 0.5 ; 0.15 ;$ zeros $(4,1)]$ and $\alpha=[-1.146+1.189 \mathrm{i} ; 1.191-0.038 \mathrm{i}]$. For SER $=10^{-3}$, once again our codes demonstrate a gain of more than $2 \mathrm{~dB}$ gain when compared with the unitary codes.

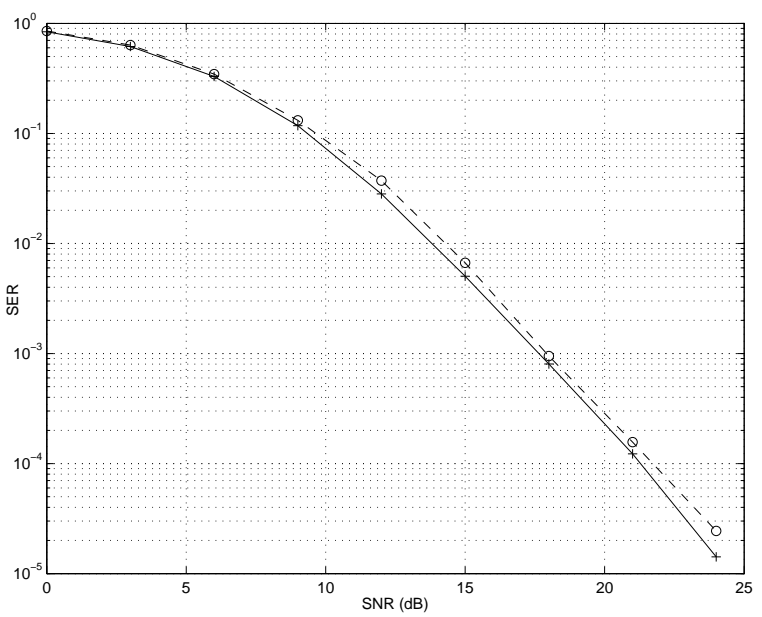

Fig. 1. Category 1 - spatio-temporally white observation noise: $T=8$,

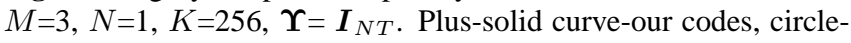
dashed curve-unitary codes. 


\begin{tabular}{|r|r||r|r|r||}
\cline { 3 - 5 } \multicolumn{1}{c||}{} & \multicolumn{3}{c|}{ PACKING RADII (DEGREES) } \\
\hline$T$ & $K$ & MB & JAT & Rankin \\
\hline 5 & 6 & 78.46 & 78.46 & 78.46 \\
\hline 5 & 7 & 74.55 & 74.52 & 75.04 \\
\hline 5 & 8 & 72.83 & 72.81 & 72.98 \\
\hline 5 & 9 & 71.33 & 71.24 & 71.57 \\
\hline 5 & 10 & 70.53 & 70.51 & 70.53 \\
\hline 5 & 11 & 69.73 & 69.71 & 69.73 \\
\hline 5 & 12 & 69.04 & 68.89 & 69.10 \\
\hline 5 & 13 & 68.38 & 68.19 & 68.58 \\
\hline 5 & 14 & 67.92 & 67.66 & 68.15 \\
\hline 5 & 15 & 67.48 & 67.37 & 67.79 \\
\hline
\end{tabular}

Table 1. PACKING IN COMPLEX PROJECTIVE SPACE: We compare our best configurations $(\mathrm{MB})$ of $K$ points in $\mathbb{P}^{T-1}(\mathbb{C})$ against the Tropp codes (JAT) and Rankin bound [5]. The packing radius of an ensemble is measured as the acute angle between the closest pair of lines.

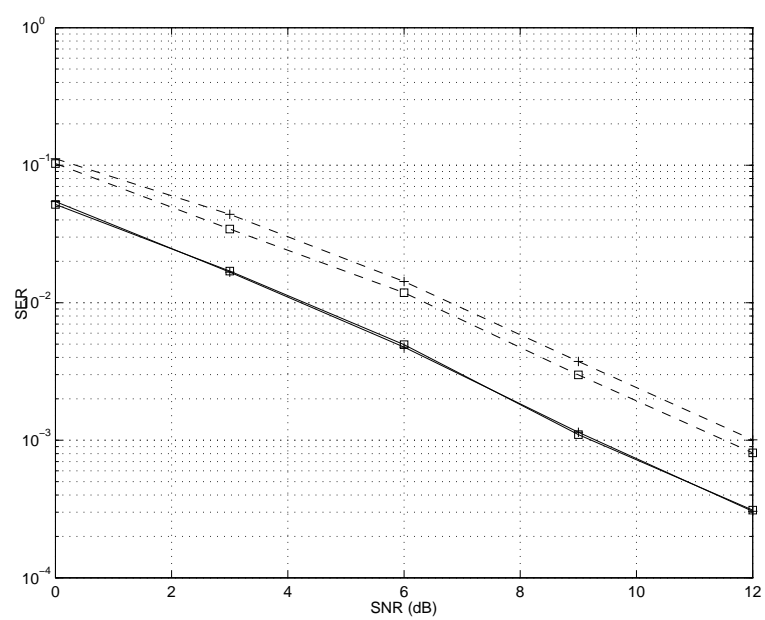

Fig. 2. Category 2 - spatially white - temporally coloured: $T=8$, $M=2, N=1, K=67, \rho=[1 ; 0.85 ; 0.6 ; 0.35 ; 0.1$; zeros $(3,1)]$. Solid curves-our codes, dashed curves-unitary codes, plus signed curvesGLRT receiver, square signed curves-Bayesian receiver.

\section{CONCLUSIONS}

We addressed the problem of codebook construction for non-coherent communication in multiple-antenna wireless systems. In contrast with other related approaches, the Gaussian observation noise may have an arbitrary correlation structure. The non-coherent receiver operates according to the GLRT principle. We proposed a methodology for designing space-time codebooks for this non-coherent setup, taking the probability of error of the detector in the high SNR regime as the code design criterion. We proposed a two-phase approach to solve the resulting high-dimensional, nonlinear and non-smooth optimization problem. Computer simulations show that our codebooks are marginally better than state-of-art known solutions for the special case of spatio-temporal white Gaussian observation noise but significantly outperform them in the correlated noise environments. This shows the relevance of the codebook construction tool proposed herein.

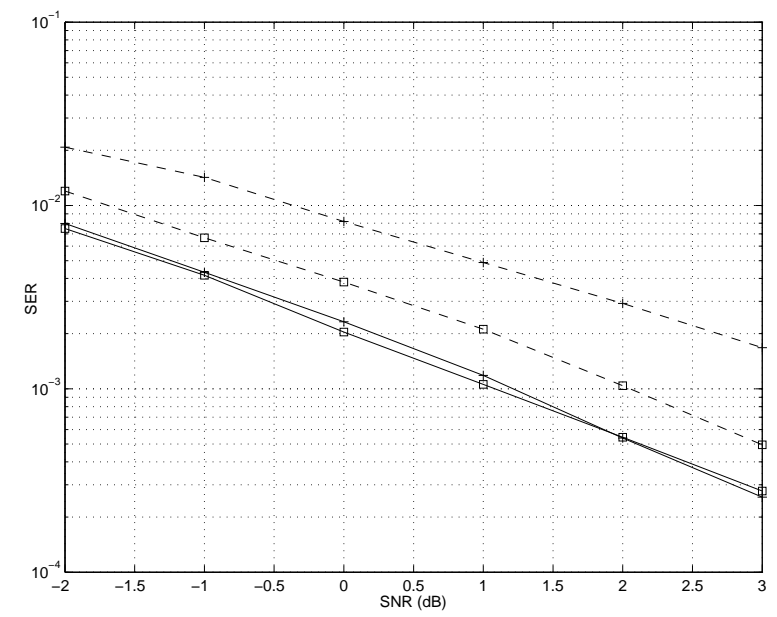

Fig. 3. Category 3: $T=8, M=2, N=2, K=32$. Solid curvesour codes, dashed curves-unitary codes, plus signed curves-GLRT receiver, square signed curves-Bayesian receiver.

\section{REFERENCES}

[1] I. Telatar, "Capacity of multi-antenna Gaussian channels," Technical Memorandum, AT\&T Bell Laboratories, 1995.

[2] T. L. Marzetta and B. M.Hochwald, "Capacity of a mobile multiple-antenna communication link in Rayleigh flat fading," IEEE Trans. Inform. Theory, vol. 45, pp. 139-157, Jan. 1999.

[3] B. M. Hochwald and T. L.Marzetta, "Unitary space-time modulation for multiple-antenna communication in Rayleigh flatfading,"IEEE Trans. Inform. Theory, vol. 46, pp. 543-564, Mar. 2000.

[4] B. M. Hochwald, T. L. Marzetta, T. J. Richardson, W. Sweldens, and R. Urbanke, "Systematic design of unitary space-time constellations," IEEE Trans. Inf. Theory, vol. 46, no. 6, pp. 1962-1973, Sep. 2000.

[5] J. A. Tropp, “Topics in sparse approximation", Ph.D. dissertation: Univ. Texas at Austin, 2004.

[6] E. G. Larsson, P. Stoica, Space-Time Block Codes for Wireless Communications., Cambridge University Press, 2003.

[7] L. L. Scharf, Statistical Signal Processing: Detection, Estimation, and Time Series Analysis., New York: Addison-Wesley Publishing Co., 1990

[8] M. Beko, J. Xavier and V. Barroso, "Non-coherent Communication in Multiple-Antenna Systems: Receiver design and Codebook construction," in preparation.

[9] J. F. Sturm, "Using SeDuMi 1.02, a MATLAB toolbox for optimization over symmetric cones (Updated for Version 1.05)," http://sedumi.mcmaster.ca

[10] M. X. Goemans, "Semidefinite programming in combinatorial optimization," Mathematical Programming, Vol. 79, pp. 143$161,1997$.

[11] A. Edelman, T. A. Arias, and S. T. Smith, "The geometry of algorithms with orthogonality constraints," SIAM J. Matrix Anal. Appl., vol. 20, no. 2, pp. 303-353, 1998.

[12] J. H. Conway, R. H. Hardin, and N. J. A. Sloane, "Packing lines, planes, etc ...: packing in Grassmannian spaces," Experiment. Math., vol. 5, pp. 139-159, Sept. 1996. 